\title{
A Multi-objective Hybrid Optimization for renewable energy integrated Electrical Power Transmission Expansion Planning
}

Original Scientific Paper

\author{
Shereena Gaffoor \\ Department of Ship Technology, \\ Cochin University of Science and Technology \\ South Kalamassery, Ernakulam, India \\ shereena.g@gmail.com \\ Mariamma Chacko \\ Department of Ship Technology, \\ Cochin University of Science and Technology \\ South Kalamassery, Ernakulam, India \\ mariamma@cusat.ac.in
}

\begin{abstract}
Due to the large size of conventional electrical power transmission systems and the large number of uncertainties involved, achieving the most favourable Transmission Expansion Planning solution turns out to be almost impossible. The proposed method intends to develop a novel method to solve Transmission Expansion Planning problems in electric power systems incorporating renewable energy sources like wind turbines and Photo Voltaic array using IEEE 24 Reliability Test System. For enhancing the efficiency of search processes and to make its use easier on diverse networks and operations, the hybridization of two renowned meta-heuristic algorithms known as Grey Wolf Optimization (GWO) and Genetic Algorithm (GA) termed as Grey Wolf with Genetic Algorithm (GWGA) is adopted. A novel distance factor based on the best position and current position of the solution in Grey wolf optimization is introduced and proposed for the hybridization technique and gives a quick and promising solution with reduced computational time. The GWO and GA algorithms are combined suitably to achieve the advantages of both algorithms. With this proposed model, the investment cost of the transmission line and the maximum amount of power that can be distributed to the consumer is optimized with an objective of minimum load shedding. Among the state-of-the-art optimization techniques considered, a remarkable performance percentage improvement in the expansion plan in terms of cost reduction and load shedding minimization has been obtained in GWO, but when hybridized with GA, an improvement of $13.42 \%$ in cost function and $18.65 \%$ in load shedding is achieved for a population size of 60 . Hence, the proposed method guarantees to generate the best solution with a faster convergence resulting in reduced computational time.
\end{abstract}

Keywords: genetic algorithm, grey wolf optimization, multi-objective, transmission planning, renewable integration

\section{INTRODUCTION}

Transmission systems exists as the foremost element of electric power production [1-2]. It not only offers an association for distribution and generation but also provides consistent and non-discriminative surroundings to consumers and suppliers. The intention of a power transmission network is to convey power from production plants to load centres efficiently, economically, reliably and securely [3-5]. As transmission systems expand, Transmission Expansion Planning (TEP) entails recognizing where to include new circuits to congregate the increased requirement by transferring the power to a new network from the old one. In the last few years, investigations in the area of transmission planning designs have seen an expansion. Several reports and publications regarding novel designs have been available in the technical literature owing to the development of novel optimization algorithms, computer power accessibility and the larger uncertainty level caused by the power sector deregulation.

Transmission system planners exploit mechanical expansion designs to find out a favourable expansion system by reducing the numerical objective functions with respect to the number of constraints. Power system planning is essential to provide reliable, sustain- 
able and affordable energy appropriately in developed countries. As a result, power system planning and associated energy issues have captured the attention of the research society [6-8].

From an upcoming scenario of power generation and requirement, the major intention of the TEP crisis solution is to discover the best set of fortifications for transmission that guarantees sufficient distribution of power to users. Therefore, the static TEP crisis solution should indicate how much and where the transmission equipment must congregate the requirements of energy. On the other hand, with the minimum feasible expenses, TEP crisis solutions should meet particular quality stipulations regarding the services. Conventionally, with the intention of guaranteeing the security criterion, TEP issues have been brought to a solution by deterministic models like renowned " $\mathrm{N}-1$ " and "N-2" [911]. In several cases, the attained arrangement can assist in the increased cost of investment.

A novel evolutionary algorithm [1] to resolve TEP issues in electric power systems was suggested to deploy various operators and a system for selecting the feasibilities of these operators. The arithmetical formulation regards a DC network design together with 'N-1'deterministic criterion and the transmission losses. The implemented technique was applied to a renowned test method and has proven its efficiency. An innovation model [7] was proposed for the planning and modelling of internally connected power systems. The suggested planning model focuses on Carbon Capture and Sequestration technologies, renewable energies and DemandSide Management as well as $\mathrm{CO} 2$ and reserve emission restraints. The innovation of this model relies on an estimation of the above-mentioned proposals which were integrated that could expose feasible synergies and interactions contained by the power system. A Constructive Heuristic Algorithm (CHA) [8] was suggested to discover the most applicable route from candidate expansion routes set for minimizing the exploration space and as a result, enlarge the effectiveness of the PSO process. The suggested methodology was practiced with two real equivalent systems and with the Garver system for the Southeast and South of Brazil in which the effectiveness of the proposed scheme can be confirmed. A preliminary Generation Expansion Planning (GEP) design [9] with obtainable input data from a variety of sources was proposed. Discrete circumstances of feasible climate change effects were described and optimization designs were configured to distinctively design uncertainty. Associations among GEP parameters and climate change were described for the entire situation to regard their effects. A multi-objective shuffled frog leaping scheme [10] was suggested to manage with the bi-level, multi-objective and non-linear nature of the design. The achieved outcomes demonstrate that the implementation of an appropriate approach for TEP could lead to more private investment in the absorption of wind power devoid of a noteworthy transmission investment cost. A proficient and enhanced GA [11] suggested was capable of functioning with various updated operators to guarantee its suitable computation in attaining constructive and best possible coordinated planning crisis solutions. A model [12] that executes optimal TEP powerfully in a Stochastic Optimization context was suggested. The design exploits a customized model of Benders' decomposition that remunerates from numerous developments that were portrayed. It manages with the integration of eventualities using a double structural design for Benders cuts and an improved contingency incorporation scheme. Additionally, it includes the capability to discover the potentially interesting candidate transmission lines mechanically which was particularly exciting in large-scale inconveniences. A static representation for Coordinated Generation and Transmission Expansion Planning (CGTEP) was proposed [13]. On minimizing the investment cost, energy and operation were not provided inside the system, the design intends to alleviate the susceptibility of a power system in opposition to physical intentional attacks in the planning horizon. In addition, the peak load of twelve days in a year was taken as a sample of the months to consider the impacts of load distinctions over a year. The physical intentional attacks and their resulting impacts were measured through the scenario building process. Accordingly, in any given month, the entire scenario was constructed as an attack plan aiming at the transmission system and therefore, they were assigned weights, which were proportional to the subsequent damage imposed on the power system. The importance of the suggested scheme in mitigating the susceptibility of power system was well established by mathematical results. A possible reason for this may be technical security solutions because they rarely include the influence of the human factor on the system security level.

Several research works on hybridized optimization of Transmission Expansion problem have been done recently. A hybrid algorithm that combines Benders decomposition and a Bees algorithm is introduced in [14] and has been tested using the transmission network expansion and energy storage planning model. The hybridized model is designed to produce good solutions quickly while still retaining a guarantee of optimality when run for a sufficiently long time. A non-linear control parameter based on cosine function is presented to replace the original linear parameter of Grey Wolf Optimizer in [15]. Also, the crossover and mutation operation of Genetic Algorithm is introduced into GWO to avoid local minima and premature convergence. A novel hybrid optimization technique based on Differential Evolution and Continuous population based Incremental Learning is proposed in [16] with load Shedding formulation as well as it includes the optimization of shunt compensation. The min conflict local search algorithm hybridized with Grey wolf optimizer for the power scheduling problem is proposed in [17]. The proposed method is compared with twenty state-of-the-art methods. Stochastic transmission expansion planning in the presence of 
wind farms considering reliability and N-1 contingency using Grey wolf Optimization technique is proposed in [18]. To check the effectiveness of the proposed method it is compared with other optimization techniques. A new hybrid GA with linear modelling is proposed for TEP Problem in [19]. It is tested on Garver 6 bus system, IEEE 24 bus and South Brazilian test which showed a rapid convergence on the test problem. A hybrid Genetic gradient algorithm is proposed in [20]. The effectiveness and practicability of the proposed model are verified by choosing a Central region of China.

This paper proposes a technique for solving the problems associated with TEP in electrical power systems incorporating renewable energy sources such as wind turbines and Photo Voltaic (PV) array using IEEE 24 Reliability Test System (RTS). For enhancing the exploitation of the transmission systems in various operating conditions and to improve the exploring efficiency, two renowned algorithms namely, Genetic Algorithm (GA) and Grey Wolf Optimization (GWO) are hybridized and adopted.

GWO has proven better capability to not fall in local optima in the initial search [21, 22]. GA can converge to get an overall good solution [23-26]. Hence an algorithm is proposed to have GWO followed by GA by including a novel distance factor for hybridization. Using this proposed GWGA algorithm, the reinforcement line has been optimized and the load has been maximized with reduced investment cost. Among the state-of-the-art optimization methods compared, GWO gives the best cost function and minimum load shedding but when hybridized with GA it gives a superior solution compared to when GWO and GA performed individually.

Next, to the implementation, this scheme is compared with the traditional algorithms such as GA, Particle Swarm Optimization (PSO), Artificial Bee Colony $(A B C)$, Firefly (FF) and GWO, and the results are presented.

The paper is organized as follows: Section 2 illustrates the TEP problem statement and Section 3 explains the proposed GWGA algorithm. Section 4 demonstrates the TEP enhancement by hybrid mechanism, Section 5 provides the results and discussion to examine the performance of the proposed methodology and Section 6 concludes the research work.

\section{TEP PROBLEM STATEMENT}

Several mathematical models for modelling TEP found in the literature are transportation model, DC model, AC model, hybrid model and disjunctive model [3-4]. These models differ from each other in the level of complexity and accuracy. In this work, DC power flow model is used as it is widely used in Transmission Expansion Planning $[27,28]$. However, the methodology presented in this work can be extended to the other models also. The formulation of the classic load flow problem $[29,30]$ requires considering four vari- ables at each bus i of the power system. These variables are the $P_{i}$ (Net active power injection), $Q_{i}$ (Net reactive power injection), $V_{i}$ (Voltage magnitude) and $\partial_{i}$ (Voltage angle).

Direct current Load flow (DCLF) gives an estimation of line power flows on AC power systems. DCLF looks only at active power flows and neglects reactive power flows. In DCLF, the non-linear model of the AC system is simplified to a linear form.

The suggested technique for the TEP crisis [31] is dependent on deterministic and static modelling [12] with single-stage production and power requirement. The TEP comprises in formatting such branches of the network which must be made stronger by integrating the required amount of reinforcement. Reinforcements are tools to be mounted on the system and could not be fractionated so that the candidate plan variable $z$ must be an integer value [12].

The actual crisis of the TEP problem is partitioned into two sub-problems [1] which are Operational Problem (OP) and Investment Problem (IP). IP is categorized as a non-linear constrained optimization problem in which the concern is to find an expansion model, which reduces the investment expenditure meeting security measures and upcoming demand.

For a specified expansion plan $z$, the objective function of the crisis is given by $g(z)$. IP is modelled by Eq. (1).

$$
\begin{array}{cl}
\text { Minimize } & g(z)=\sum_{(i, j) \in \mu} C_{i j} n_{i j} \\
& B(z)=0 \\
\text { Subject to } & C(z)=0 \\
& 0 \leq n_{i j} \leq n_{i j \max }
\end{array}
$$

$C_{i j}$ is the unit cost related to the reinforcements to branch connecting bus $i$ to $j, \mu$ indicates the group of entire candidate branches of the system network, $n_{i j \max }$ is the maximum number of reinforcements to be integrated to branch connecting bus $i$ to $j$. Function $B(z)$ indicating load shedding and $C(z)$ indicating overload are described subsequently.

For a specified expansion plan $z$, the OP is considered as a problem of LP, where the objective is to find the economic transmit of generators which reduces the total load shedding $B(z)$ and is given by Eq. (2).

$$
\begin{array}{ll}
\text { Minimize } & \multicolumn{1}{c}{B(z)=\sum_{p=1}^{\alpha} r_{p}} \\
& f+r+A \theta=h \\
& \left|g_{i j}\right| \leq\left(n_{i j}^{0}+n_{i j \max }\right) g_{i j \max } \\
\text { Subject to } & f_{\min } \leq f \leq f_{\max } \\
& 0 \leq r \leq h
\end{array}
$$


The amount of generation produced by renewable energy sources (Wind turbine and PV array) are represented by $r$ whose constituent $r_{p}$ indicates the load shed at bus $p$.

Hence $r_{p}$ can be represented by Eq. (3).

$$
r_{p}=\left\{\begin{array}{ll}
r^{w} & \text { if } R E=1 \\
r^{p v} & \text { if } R E=2
\end{array}\right\}
$$

where $R E$ refers to renewable energy sources, $r^{w}$ indicates energy from wind turbine, $r^{p w}$ indicates energy from PV array.

Moreover, the generating vector is indicated by $f$ and its constituent $f_{b}$ denotes the production at bus $b$, the network susceptance matrix is denoted by $A$, the vector of load demand is given by $h$ where its constituent $h_{p}$ denotes the load at bus $p$, the voltage angle vector is denoted by $\theta$ and its constituent $\theta_{p}$ indicates the angle at bus $p, g_{i j}$ is the active power flow of the branch connecting bus $i$ to $j$ given by $g_{i j}=\left(\theta_{i}-\theta_{j}\right) / x_{i j}$ and $x_{i j}$ denotes the reactance. The maximum capacity of each constituent is represented by $g_{i j \max }$ for branch connecting bus $i$ to $j$; the $\operatorname{term} f_{\max }$ and $f_{\min }$ denote the vectors that symbolise the maximum and minimum capacity of generators; the number of elements in the branch connecting bus $i$ to $j$ at the base case is indicated by $n^{0}{ }_{i j}$ and $\alpha$ is the group of entire network buses. The values of $f, r, h, g$ and $x_{i j}$ are given in p.u and $\theta$ values are denoted in radians.

Overload function is represented by $C(z)$. The value of $C(z)$ is obtained by solving DC power flow using the generator vector found by the OP solution. For each branch of the system, the occurrence of overloads is verified. Thus overload function will depend on the reinforcements present in the plan $z$ and should be nil.

The total energy from wind turbine $r^{w}$ which is generated depending on the texture speed [32] is given by Eq. (4).

$$
r^{w}=\sum_{s=1}^{\omega} \frac{M_{0} D v_{s}^{3} L_{p}}{2 O_{L} T} e^{\frac{-g H}{O_{L} T}}
$$

$\omega$ indicates the number of wind turbines, $M_{0}$ is the standard sea-level atmospheric pressure (101325 Pa), $D$ is the swept area in $\mathrm{m}^{2}, v$ indicates velocity of the wind turbine in $\mathrm{m} / \mathrm{s}, L_{p}$ is the wind turbine power coefficient defined as the ratio of actual power produced by a wind turbine divided by the total wind turbine power flowing into the turbine blades, $O$ indicates specific gas constant for air $(287 \mathrm{~J} /(\mathrm{Kg} . \mathrm{K})), T$ is the temperature in Kelvin which can be computed at any height in meter as $T=T_{0^{-}}-L H$, where $T_{0}$ is the temperature at sea level $(288 \mathrm{~K}), L$ is the temperature lapse rate $\left(0.0065^{\circ} \mathrm{C} / \mathrm{m}\right)$, $H$ is the altitude above sea level in $\mathrm{m}, g$ is the gravity constant $(9.8 \mathrm{~m} / \mathrm{s} 2)$.

The electrical energy generated from the PV array [33] can be found out as given in Eq. (5).

$$
r^{P V}=\sum_{t=1}^{q} \eta_{m} C_{m} I(t)
$$

For the proposed research work, identical PV modules are considered. Here $\eta_{m}$ indicates PV module efficiency, $C_{m}$ indicates area of PV module in $\mathrm{m}^{2}, I(t)$ refers to solar intensity $\left(\mathrm{W} / \mathrm{m}^{2}\right)$ and $q$ is the number of PV modules.

\section{PROPOSED GWGA ALGORITHM}

The conventional Grey Wolf Optimization algorithm has certain disadvantages such as low solving precision, slow convergence and bad local searching ability. In order to overcome these disadvantages of GWO, it is hybridized with GA algorithm, as it can provide global optimal solutions. By hybridizing both algorithms, the solution for minimizing the investment cost with the objective function can be obtained.

The process of GWO algorithm [34-35] describes the hierarchy of grey wolves hunting and leadership characteristics. There are four classifications of grey wolves known as $a, \beta, \omega$ and $\delta$ that are exploited for executing the leadership hierarchy. Penetrating, encircling and attacking the prey are the three chief practices in hunting that are deployed to progress optimization.

A genetic algorithm [36-37] is a search heuristic that is inspired by Charles Darwin's theory of natural evolution. This algorithm reflects the process of natural selection where the fittest individuals are selected for reproduction in order to produce offspring of the next generation. GAs are the ways of solving problems by mimicking processes that nature uses, i.e., Selection, Crossover, Mutation and accepting to evolve a solution to a problem.

GWGA algorithm is the hybridization of GWO and GA algorithms. Here, the crossover rate $r_{c}$ is fixed at 0.6, and the bounding factor is given by $b$ as in Eq. (6), where $b_{\max }$ and $b_{\min }$ indicates the upper bound and lower limits of the solution. Moreover, a distance factor ' $d$ ' based on the best position and current position of solution is introduced and can be evaluated as given by Eq. (7), where $\alpha_{p o}$ is the best position of the solution and $\varsigma_{p o}$ is the current position of the solution.

$$
\begin{aligned}
& b=\sqrt{\operatorname{mean}\left(b_{\max }-b_{\min }\right)^{2}} \\
& d=\sqrt{\operatorname{mean}\left(\alpha_{p o}-\varsigma_{p o}\right)^{2}}
\end{aligned}
$$

Moreover, the distance threshold $d_{t h}$ can be evaluated by Eq. (8), where Iter denotes current iteration and Iter $_{\max }$ indicates the maximum iteration.

$$
d_{t h}=r_{c} \times b \times \frac{\text { Iter }}{\text { Iter }_{\text {max }}}
$$

Also, if the distance $d$ is greater than $d_{t h}$ the solution can be updated using GWO. Otherwise, crossover operation has to be performed using the principle of GA algorithm, and the updated solution can be obtained as shown in Eq. (9).

$$
Z_{2}^{*}=\frac{\text { child } 1+\text { child } 2}{2}
$$


Thus, the optimized solution of TEP is obtained as $Z^{*}$, which attain the minimized cost and balanced power generation in order to meet the demand of the power system.

The flow chart for the proposed GWGA algorithm is shown in Fig. 1

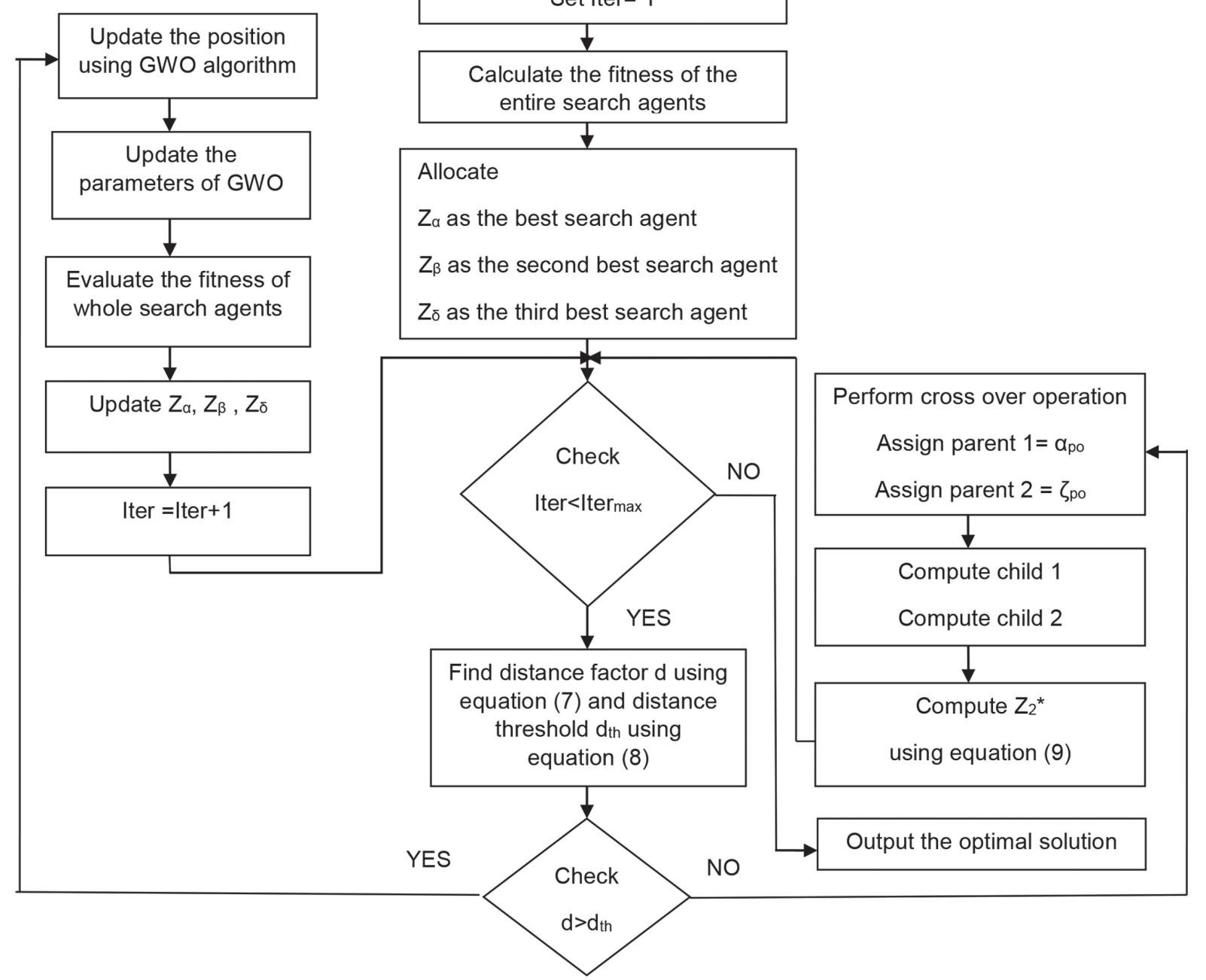

Fig. 1.Flowchart of proposed GWGA Algorithm

\section{TEP ENHANCEMENT BY HYBRID OPTIMIZATION}

For solving the TEP problem, the number of branches, generators and renewable energy sources is given as solutions for encoding, which has to be optimized. The bounding limit of the number of branches ranges from $n_{i j \min }$ to $n_{i j \max }$. Similarly, the bounding limit of generator bus ranges from $g_{i j \min }$ to $g_{i j \max }$ and the bounding limit of renewable energy sources ranges from 0 to 2, i.e. $R E_{i} \in\{0,1,2\}$. If $R E_{i}=0$, it means that there were no renewable energy sources connected to the bus. If $R E_{i}=1$, the wind turbine is connected to the bus and if $R E_{i}=2$, PV array is connected to the bus system. Fig. 2 reveals the solution given for encoding process, where $N_{B}$ indi- cates the number of branches, $N_{G}$ denotes the number of generators and $N_{R}$ is the count of renewable sources connected to the IEEE 24 bus systems. The length of the solution is the summation of $N_{B}, N_{G}$ and $N_{R}$. Collectively, the solution that is to be optimized is termed as $Z$.

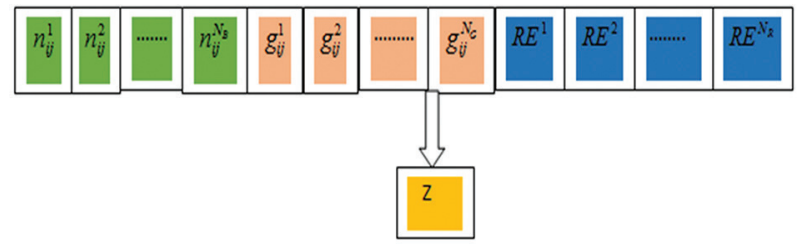

Fig. 2. TEP solution encoding 
The IEEE 24 RTS shown in Fig. 3 includes 11 synchronous generators with 17 load points and 38 branches, where each branch can receive a maximum of three reinforcements; the total demand is $2850 \mathrm{MW}$ and the maximum generation capacity is $3405 \mathrm{MW}$.

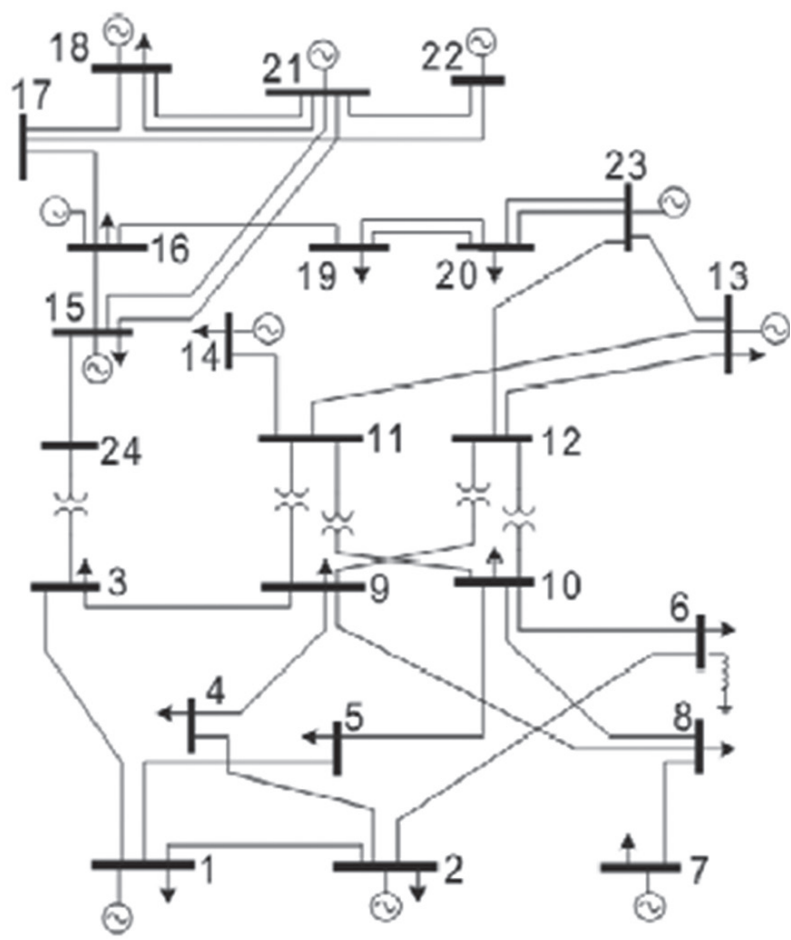

Fig. 3. Single line diagram of IEEE 24 bus Reliability Test System

Table 1 gives the transmission line data indicating the branch numbers. For the optimization process the length of the chromosome (solution size) is taken as 61. Chromosome number 1 to 38 indicate the number of branches, 39 to 47 represents the nodes where generators are connected and 48 to 61 represents the nodes where renewable energy sources are connected.

All wind farms are equipped with three $1.5 \mathrm{MW}$ wind turbines. Table 2 shows the manufacturer only specification of a 1.5 MW GE wind turbine [32].

Various design parameters taken for calculating the electrical energy from PV array are: $\mathrm{Am}=1.3264 \mathrm{~m}^{2}$, $\eta_{\mathrm{m}}=15 \%$ and $\mathrm{I}(\mathrm{t})=1000 \mathrm{~W} / \mathrm{m}^{2}[33]$.

\section{RESULTS AND DISCUSSIONS}

The proposed TEP model with renewable energy sources [38-40] such as wind turbine and PV array using GWGA method has been simulated in MATLAB, tested in IEEE 24 bus system and the results obtained are analysed. The performance of the proposed model has been compared with traditional algorithms such as GA [23-26], PSO [41-46], ABC [47-48], FF [49-50] and GWO [22-28] based on cost function and load shedding function. The corresponding outcomes were evaluated to certify the performance of the proposed model.
Table 1. Transmission line data indicating branch number

\begin{tabular}{|c|c|c|c|c|c|}
\hline Line No. & $\begin{array}{c}\text { From } \\
\text { Bus No. }\end{array}$ & $\begin{array}{l}\text { To Bus } \\
\text { No. }\end{array}$ & Line No. & $\begin{array}{c}\text { From } \\
\text { Bus No. }\end{array}$ & $\begin{array}{c}\text { To Bus } \\
\text { No. }\end{array}$ \\
\hline 1 & 1 & 2 & 20 & 12 & 13 \\
\hline 2 & 1 & 3 & 21 & 12 & 23 \\
\hline 3 & 1 & 5 & 22 & 13 & 23 \\
\hline 4 & 2 & 4 & 23 & 14 & 16 \\
\hline 5 & 2 & 6 & 24 & 15 & 16 \\
\hline 6 & 3 & 9 & 25 & 15 & 21 \\
\hline 7 & 3 & 24 & 26 & 15 & 21 \\
\hline 8 & 4 & 9 & 27 & 15 & 24 \\
\hline 9 & 5 & 10 & 28 & 16 & 17 \\
\hline 10 & 6 & 10 & 29 & 16 & 19 \\
\hline 11 & 7 & 8 & 30 & 17 & 18 \\
\hline 12 & 8 & 9 & 31 & 17 & 22 \\
\hline 13 & 8 & 10 & 32 & 18 & 21 \\
\hline 14 & 9 & 11 & 33 & 18 & 21 \\
\hline 15 & 9 & 12 & 34 & 19 & 20 \\
\hline 16 & 10 & 11 & 35 & 19 & 20 \\
\hline 17 & 10 & 12 & 36 & 20 & 23 \\
\hline 18 & 11 & 13 & 37 & 20 & 23 \\
\hline 19 & 11 & 14 & 38 & 21 & 22 \\
\hline
\end{tabular}

Table 2. Specification of a GE 1.5SLE wind turbine

\begin{tabular}{|cc|}
\hline Parameter & Value \\
\hline Rated Output & $1.5 \mathrm{MW}$ \\
\hline Rotor diameter & $77 \mathrm{~m}$ \\
\hline Cut in wind speed & $3.5 \mathrm{~m} / \mathrm{s}$ \\
\hline Rated wind speed & $14 \mathrm{~m} / \mathrm{s}$ \\
\hline Cut out wind speed & $25 \mathrm{~m} / \mathrm{s}$ \\
\hline Hub height & $80 \mathrm{~m}$ \\
\hline
\end{tabular}

\subsection{COST FUNCTION ANALYSIS}

The performance comparison of the cost function of the proposed GWGA model with conventional algorithms is given in Fig. 4. For evaluating the effectiveness of the solution, i.e. to check whether the solution is local optimal or global optimal, the number of iterations is extended to 500 for a population size of 10 and the performance is shown in Fig. 5. The unit cost is randomly generated for the given population size. The proposed method shows the effectiveness in reducing the computational time and hence shows better convergence speed. 


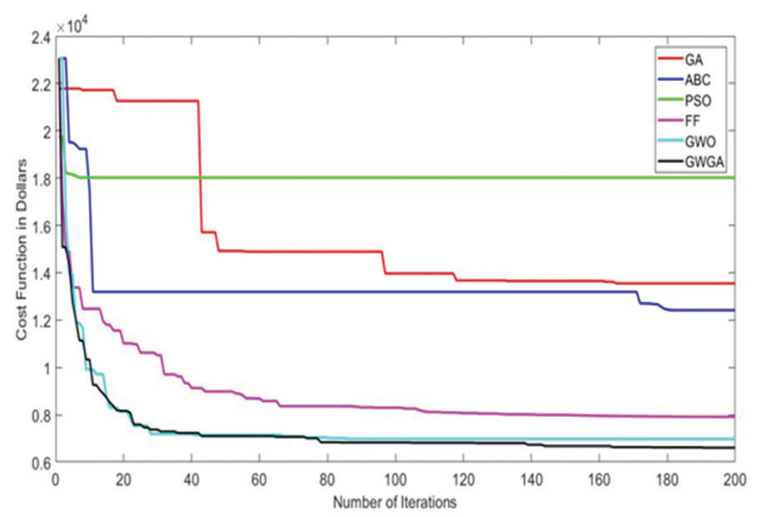

Fig. 4. Cost function analysis of different optimization techniques for iterations 0 to 200

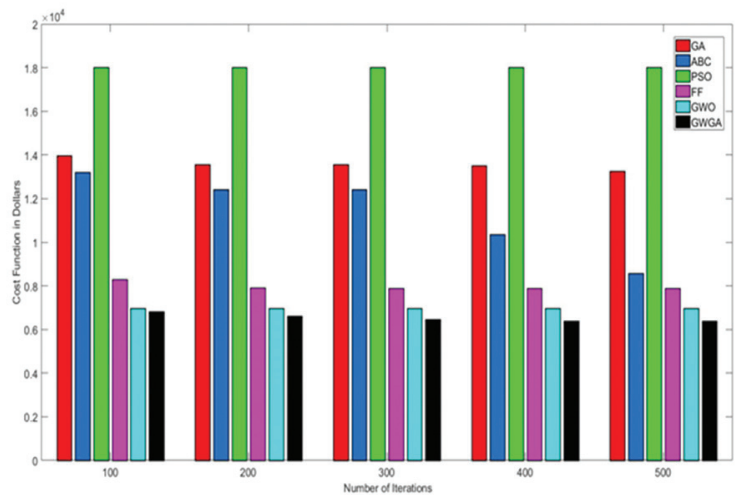

Fig. 5. Cost function analysis of different optimization techniques for iterations 100 to 200

The performance analysis is also done for varying population sizes $10,30,60,80$ and 100 for 200 iterations and the comparison result is shown in Table 3 and the performance improvement percentage of the proposed GWGA method compared with conventional algorithms is given in Table 4.

The results show that the proposed GWGA gives better performance than the state-of-the-art optimization methods compared in the proposed work in TEP. For all the population size considered, GWO gives the best performance and the quality solution is obtained for a population size of 100 . When hybridized with GA, the best solution is obtained for a population size 60 giving a $15.76 \%$ improvement in cost function than the individual approach. The result also shows that the best solution obtained with GWGA is $13.42 \%$ better than the best solution obtained for a population size 100 when GWO performed individually. Similarly, with GA approach, the best solution is obtained for a population size of 100 . When hybridized with GWO, the best solution is obtained for a population size 60 giving a $61.95 \%$ improvement in cost function than the individual approach. The result also shows that the best solution obtained with GWGA is $54.42 \%$ better than its best solution when GA has been done individually. Hence the proposed hybridization gives the best solution for less population size thereby computation time is reduced.

The results also indicate the effectiveness of the proposed method to deal with different sizes of the network and hence the proposed method is scalable. Thus, from the simulation analysis, the functionality of the proposed scheme to meet the considered power demand with minimal cost can be attained.

Table 3. Cost function analysis using different optimization techniques with varying population size

\begin{tabular}{|ccccccc|}
$\begin{array}{c}\text { Population } \\
\text { Size }\end{array}$ & $\mathbf{1 0}$ & $\mathbf{3 0}$ & $\mathbf{6 0}$ & $\mathbf{8 0}$ & $\mathbf{1 0 0}$ \\
\hline GA & 15795.9 & 16486.9 & 17050.68 & 14957.14 & 14235.5 \\
\hline ABC & 10229.5 & 12221.01 & 13512.73 & 12673.2 & 10908.36 \\
\hline PSO & 18492.77 & 17738.34 & 18137.93 & 14957.14 & 14235.5 \\
\hline FF & 9385.805 & 15187.47 & 12271.21 & 9566.683 & 13014.52 \\
\hline GWO & 7782.325 & 7826.439 & 7701.398 & 7764.962 & 7492.607 \\
\hline GWGA & 7197.423 & 7197.894 & 6487.372 & 6493.273 & 7233.44 \\
\hline
\end{tabular}

Table 4. Performance Improvement of GWGA with respect to conventional algorithms (\%)

\begin{tabular}{cccccc|}
$\begin{array}{c}\text { Population } \\
\text { Size }\end{array}$ & $\mathbf{1 0}$ & $\mathbf{3 0}$ & $\mathbf{6 0}$ & $\mathbf{8 0}$ & $\mathbf{1 0 0}$ \\
\hline GA & 54.43 & 56.34 & 61.95 & 56.59 & 49.19 \\
\hline ABC & 29.64 & 41.1 & 51.99 & 48.76 & 33.69 \\
\hline PSO & 61.08 & 59.42 & 64.23 & 56.59 & 49.19 \\
\hline FF & 23.32 & 52.61 & 47.13 & 32.13 & 44.42 \\
\hline GWO & 7.52 & 8.03 & 15.76 & 16.38 & 3.46 \\
\hline
\end{tabular}

\subsection{LOAD SHEDDING ANALYSIS}

The performance comparison of load shedding of the proposed GWGA model with conventional algorithms is given in Fig. 6 . The number of iterations is extended to 500 for a population size of 10 giving the convergence speed performance as shown in Fig. 7.

The performance analysis is also done for varying population sizes $10,30,60,80$ and 100 for 200 iterations and the comparison result is shown in Table 5 and the percentage performance improvement in load shedding of the proposed GWGA methodology compared with conventional algorithms is shown in Table 6 .

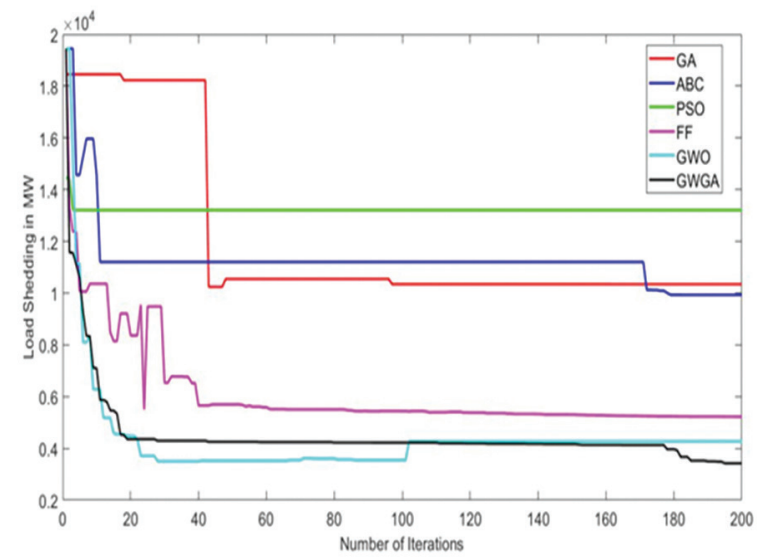

Fig. 7. Load shedding analysis of different optimization techniques for iterations 100 to 500 
Table 5. Load shedding analysis using different optimization techniques with varying population size

\begin{tabular}{cccccc}
$\begin{array}{c}\text { Population } \\
\text { Size }\end{array}$ & $\mathbf{1 0}$ & $\mathbf{3 0}$ & $\mathbf{6 0}$ & $\mathbf{8 0}$ & $\mathbf{1 0 0}$ \\
\hline GA & 10345.05 & 10913.29 & 11228.2 & 9279.316 & 8907.103 \\
\hline ABC & 5597.098 & 7674.407 & 8855.18 & 8391.756 & 6657.085 \\
\hline PSO & 13206.99 & 12081.91 & 12193.9 & 9279.316 & 8907.103 \\
\hline FF & 5201.581 & 9837.438 & 7143.93 & 4780.685 & 7857.024 \\
\hline GWO & 4269.435 & 4339.245 & 4163.62 & 4260.662 & 4215.924 \\
\hline GWGA & 4097.052 & 4097.522 & 3387 & 3392.901 & 4100.289 \\
\hline
\end{tabular}

Table 6. Performance Improvement of GWGA with respect to conventional algorithms (\%)

\begin{tabular}{|cccccc|}
$\begin{array}{c}\text { Population } \\
\text { Size }\end{array}$ & $\mathbf{1 0}$ & $\mathbf{3 0}$ & $\mathbf{6 0}$ & $\mathbf{8 0}$ & $\mathbf{1 0 0}$ \\
\hline GA & 60.4 & 62.45 & 69.83 & 63.44 & 53.97 \\
\hline ABC & 26.8 & 46.61 & 61.75 & 59.57 & 38.41 \\
\hline PSO & 68.98 & 66.09 & 72.22 & 63.44 & 53.97 \\
\hline FF & 21.23 & 58.35 & 52.59 & 29.03 & 47.81 \\
\hline GWO & 4.04 & 5.57 & 18.65 & 20.37 & 2.74 \\
\hline
\end{tabular}

For all the population size considered, GWO gives the best performance and the quality solution is obtained for a population size of 60 . When hybridized with GA, the best solution is obtained for a population size 60 giving an $18.65 \%$ improvement in load shedding function than the individual approach. Similarly, with the GA approach, the best solution is obtained for a population size of 100 . When hybridized with GWO, the best solution is obtained for a population size 60 giving a $69.83 \%$ improvement in load shedding than the individual approach. The result also shows that the best solution obtained with GWGA is $61.97 \%$ better than its best solution when GA has been done individually. Hence the proposed hybridization gives the best solution for less population size thereby computation time is reduced.

When compared with $A B C$, FF and PSO, GWGA gave the best performance for all the population size considered. Hence, the proposed method gives the best solutions of IEEE 24 bus system for load shedding analysis for a population size of 60 . The results depicted in Table 4 and Table 6 shows that GWGA converges much faster than GWO and GA algorithms. The computational time of the proposed method is less and gives the best results in the population size in the range of 30 to 60.

Table 7 shows the final expansion plan obtained by the methodology proposed. Table 8 compares the generation capacity obtained before and after TEP with GWGA methodology at different nodes and Table 9 shows the nodes where the renewable energy sources [51-55] are connected for optimal operation of the proposed work.
Table 7. Final Expansion Plan for IEEE 24 bus using the proposed methodology

\begin{tabular}{|c|c|c|c|c|c|}
\hline Line No. & $\begin{array}{c}\text { From } \\
\text { Bus No. }\end{array}$ & $\begin{array}{c}\text { To Bus } \\
\text { No. }\end{array}$ & Line No. & $\begin{array}{c}\text { From } \\
\text { Bus No. }\end{array}$ & $\begin{array}{c}\text { To Bus } \\
\text { No. }\end{array}$ \\
\hline 1 & 3 & 20 & 2 & 12 & 13 \\
\hline 2 & 2 & 21 & 2 & 12 & 23 \\
\hline 3 & 2 & 22 & 1 & 13 & 23 \\
\hline 4 & 2 & 23 & 2 & 14 & 16 \\
\hline 5 & 3 & 24 & 3 & 15 & 16 \\
\hline 6 & 1 & 25 & 1 & 15 & 21 \\
\hline 7 & 2 & 26 & 2 & 15 & 21 \\
\hline 8 & 3 & 27 & 1 & 15 & 24 \\
\hline 9 & 2 & 28 & 3 & 16 & 17 \\
\hline 10 & 2 & 29 & 3 & 16 & 19 \\
\hline 11 & 3 & 30 & 2 & 17 & 18 \\
\hline 12 & 2 & 31 & 2 & 17 & 22 \\
\hline 13 & 2 & 32 & 3 & 18 & 21 \\
\hline 14 & 1 & 33 & 2 & 18 & 21 \\
\hline 15 & 3 & 34 & 1 & 19 & 20 \\
\hline 16 & 3 & 35 & 1 & 19 & 20 \\
\hline 17 & 1 & 36 & 2 & 20 & 23 \\
\hline 18 & 3 & 37 & 3 & 20 & 23 \\
\hline 19 & 1 & 38 & 3 & 21 & 22 \\
\hline
\end{tabular}

Table 8. Comparison of Generation capacity before and after TEP at different nodes

\begin{tabular}{|ccc|}
\hline Generator Bus No. & $\begin{array}{c}\text { Generation } \\
\text { Capacity before } \\
\text { TEP without GWGA } \\
\text { (MW) }\end{array}$ & $\begin{array}{c}\text { Generation } \\
\text { Capacity after TEP } \\
\text { with GWGA (MW) }\end{array}$ \\
\hline 2 & 67 & 140.5605 \\
\hline 7 & 64 & 148.5388 \\
\hline 13 & 200 & 554.2946 \\
\hline 15 & 274 & 24.0616 \\
\hline 16 & 245 & 71.6325 \\
\hline 21 & 144 & 351.6817 \\
\hline 22 & 294 & 315.4005 \\
\hline 23 & 150 & 300 \\
\hline
\end{tabular}

Table 9. Node numbers of buses where renewable sources are connected

\begin{tabular}{c|ccccccccc}
$\begin{array}{c}\text { Types of renewable Energy } \\
\text { Sources }\end{array}$ & \multicolumn{5}{c}{ Node number } \\
\hline $\begin{array}{c}\text { Wind turbines } \\
\text { PV array }\end{array}$ & 8 & 9 & 10 & 11 & 14 & 17 & 19 \\
\hline
\end{tabular}




\section{CONCLUSION}

This paper has presented a novel scheme to resolve the issues residing in TEP of electric power systems with renewable energy sources such as wind turbines and PV array by optimizing the reinforcement line, transmit of generators and renewable energy sources. It was applied and simulated in IEEE 24 test bus system. In addition, with the intention of improving the effectiveness of transmission in diverse networks and operation conditions, the hybridization of two well-known metaheuristic algorithms said to be GWO and GA named as GWGA is adopted. In order to achieve the advantages of both the methods and obtain a faster convergence at the same time, they are combined suitably and used. Consequently, with this novel hybridized model, the investment cost of the transmission line and the maximum amount of power that can be extended to the consumer is optimized.

The proposed scheme has been compared with the conventional algorithms such as GA, PSO, ABC, FF and GWO and the results are tabulated. The proposed GWGA method shows better optimization results considering expansion cost minimization and minimum load shedding when GWO and GA optimization methods are performed individually. The method is scalable in terms of the size of the network. The GWGA gives the best solution in the population range of 30 to 80 for 200 iterations, which reduces the computation time. The GWGA appears to be a very effective hybrid algorithm for highly complex transmission expansion problems.

In further studies, the proposed method will be applied for AC analysis as well as to a real power system with real data considering uncertainties.

\section{REFERENCES:}

[1] A. M. L. da Silva, M. R. Freire, L. M. Honorio, "TEP optimization by adaptive multi-operator evolutionary algorithms", Electric Power Systems Research, Vol. 133, 2016, pp. 173-181.

[2] R. Romero, A. Monticelli, "A Zero-One Implicit Enumeration Method for Optimizing Investments in Transmission Expansion Planning", IEEE Transactions on Power Systems, Vol. 9, 1994, pp. 1385-1391.

[3] M. V. F. Pereira, L. M. V. G. Pinto, S. H. F. Cunha, G. C. Oliveira, "A Decomposition approach to automated generation/transmission expansion planning", IEEE Transactions on Power Apparatus and Systems, Vol. 104,1985, pp. 3074-3083.

[4] T. Sum-Im, W. Ongsakul, "A Self-Adaptive Differential Evolution Algorithm for Transmission Network Expansion Planning with System Losses Consid- eration", Proceedings of the IEEE International Conference on Power and Energy, Kota Kinabula, Malaysia, 2-5 December 2012, pp. 152-156.

[5] C.Tian, X. Lu, L. Chu, T. Dong, D. Li, “Multi-Objective Transmission Network Planning with Consideration of Power Grid Vulnerability and Wind Power Accommodation", Journal of Engineering Science and Technology Review, Vol. 3, 2013, pp. 30-34.

[6] M. J. Rider, A. V. Garcia, R. Romero, "Power system transmission network expansion planning using AC model", IET Generation Transmission and Distribution, Vol. 5, 2007, pp. 731-742.

[7] O. J. Guerra, D. A. Tejada, G. V. Reklaitis, "An optimization framework for the integrated planning of generation and transmission expansion in interconnected power systems", Applied Energy, Vol. 170, 2016, pp. 1-21.

[8] I. M. de Mendonca, I. C. Silver Junior, A. L. M. Marcato, "Static planning of the expansion of electrical energy transmission systems using particle swarm optimization", International Journal of Electrical Power and Energy Systems, Vol. 60, 2014, pp. 234-244.

[9] S. Li, D. W. Coit, F. Felder, "Stochastic optimization for electric power generation expansion planning with discrete climate change scenarios", Electric Power Systems Research, Vol. 140, 2016, pp. 401-412.

[10] M. Jadidoleslam, A. Ebrahimi, Mohammad Amin Latify, "Probabilistic TEP to maximize the integration of wind power", Renewable Energy, Vol. 114, 2017, pp. 866-878.

[11] H. K. Rad, Z. Moravej, "An approach for simultaneous distribution, sub-transmission and transmission networks expansion planning", International Journal of Electrical Power and Energy Systems, Vol. 19, 2017, pp. 166-182.

[12] S. Lumbreras, A. Ramos, F. Banez-Chicharro, “Optimal transmission network expansion planning in real-sized power systems with high renewable penetration", Electric Power Systems Research, Vol. 149, 2017, pp. 76-88.

[13] H. Nemati, M. A. Latify, G. R. Yousefi, "Coordinated generation and TEP for a power system under physical deliberate attacks", International Journal 
of Electrical Power and Energy Systems, Vol. 96, 2018, pp. 208-221.

[14] C. A. G. MacRae, M. Ozlen, A. T. Ernst, "The BeeBenders Hybrid Algorithm with application to Transmission Expansion Planning", Proceedings of the Genetic and Evolutionary Computation Conference Companion, Lille, France, 10-14 July 2021, pp. $1275-1282$.

[15] W. Gai, C. Qu, J. Liu, J. Zhang, "An Improved Grey Wolf Algorithm for Global Optimization", Proceedings of the $30^{\text {th }}$ Chinese Control and Decision Conference, Shenyang, China, 9-11 June 2018, pp. 2494-2498.

[16] E. G. Morquecho, S. P. Torres, N. E. Matute, Fabian Astudillo-Salinas, Julio C. Lopez, Wilfredo C. Flores, "AC Dynamic Transmission Expansion Planning using Hybrid Optimization Algorithm", Proceedings of the IEEE PES Innovative Smart Grid Technologies, The Hague, Netherlands, 26-28 October 2020, pp. 499-503.

[17] S. N. Makhadmeh, A. T. Khader, M. A. Al-Betar, S. Nain, A. K. Abasi, Z. A. A. Alyasseri, "A novel hybrid grey wolf optimizer with min conflict algorithm for power scheduling problem in a smart home", Swarm and Evolutionary Computation, Vol. 60, 2021, pp. 2210-2227.

[18] A. A. Ghadimi, M. Amami, M. Bayat, S. Ahmadi, M. R. Mireh, F. Jurado, "Stochastic Transmission Expansion Planning in the presence of wind farms considering reliability and $\mathrm{N}-1$ contigency using Grey Wolf Optimization Technique", Electrical Engineering, 2021, https://doi.org/10.007/s00202021-01339-w

[19] E. S. Yigit, S. Mutlu, B. Babayigit, "Transmission Expansion Planning based on a hybrid genetic algorithm approach under uncertainty", Turkish Journal of Electrical Engineering and Computer Science, Vol. 27, 2019, pp. 2922-2937.

[20] L. Ma, Y. Wu, S. Lou, S. Lang, M. Liu, Y. Gao, “Coordinated Generation and Transmission Expansion Planning with High Proportion of Renewable Energy", Proceedings of the $4^{\text {th }}$ IEEE Conference on Energy Internet and Energy System Integration, Wuhan, China, 30 October-1 November, 2020, pp. 1019-1024.
[21] A J. Wang, S. Li, "An Improved Grey Wolf Optimizer Based on Differential Evolution and Elimination Mechanism", Scientific Reports, Vol. 9, 2019, pp. 1-24.

[22] Z. Gao, J. Zhao, "An Improved Grey Wolf Optimization Algorithm with Variable Weights", Hindawi Computational Intelligence and Neuroscience, Vol. 2019, 2019, pp. 1-13.

[23] M. Nikolic, J. Jovic, "Implementation of genetic algorithm in map-matching model", Expert Systems with Applications, Vol. 72, 2017, pp. 283-292.

[24] R. A. Gallego, A. Monticelli, R. Romero, "Transmission system expansion planning by an extended genetic algorithm", IEE Proceedings Generation, Transmission and Distribution, Vol. 145, 1998, pp. 329-335.

[25] H. A. Gil, E. L. Silva, "A reliable approach for solving the transmission network expansion planning problem using genetic algorithms", Electric Power Systems Research, Vol. 58, 2001, pp. 45-51.

[26] Luis A. Gallego, Marcos J. Rider, Marina Lavorato, Antonio Paldilha-Feltrin, "An Enhanced Genetic Algorithm to Solve the Static and Multistage Transmission Network Expansion Planning", Journal of Electrical and Computer Engineering, Vol. 2012, 2012, pp. 1-12.

[27] G. Latorre, R. D. Cruz, J. M. Areiza, A. Villegas, "Classification of publications and models on transmission expansion planning", IEEE Transactions on Power Systems, Vol. 18, 2003, pp. 938-946.

[28] I. J. Silva, M. J. Rider, R. Romero, A. V. Garcia, C. A. Murari, "Transmission network expansion planning with security constraints", IEEE Proceedings on Generation, Transmission and Distribution, Vol. 152,2005 , pp. 828-836.

[29] Jizhong Zhu, "Optimal Power Flow", Optimization of Power System Operation, pp. 297-364, A John Wiley and Sons, Inc., Publication, 2009.

[30] Ahmed A. Fathy, Mohamed S. Elbages, Ragab A. El-Sehiemy, Fahmy M. Bendary, "Static transmission expansion planning for realistic networks in Egypt", Electric Power Systems Research, Vol. 151, 2017, pp. 404-418.

[31] M. S. El-bages, W. T. Elsayed, "Social spider algorithm for solving the transmission expansion 
planning problem", Electric Power Systems Research, Vol. 143, 2017, pp. 235-243.

[32] H. M. K. Al-Masri, M. Ehsani, "Impact of wind turbine modeling on a renewable energy system", Proceedings of the North American Power Symposium, Denver, CO, USA, 18-20 September, 2016, pp. 1-6.

[33] Sumit Tiwari, Jasleen Bhatti, G. N. Tiwari, I. M. AlHelal,"Thermal modelling of photovoltaic thermal (PVT) integrated greenhouse system for biogas heating", Solar Energy, Vol. 136, 2016, pp. 639-649.

[34] Seyedali Mirjalili, Seyed Mohammad Mirjalili, Andrews Lewis, "Grey Wolf Optimizer", Advances in Engineering Software, Vol. 69, 2014, pp. 46-61.

[35] Attia A. El-Fergany, Hany M. Hasanien, "Single and Multi-objective Optimal Power Flow Using Grey Wolf Optimizer and Differential Evolution Algorithms", Electric Power Components and Systems, Vol. 43, 2015, pp. 1548-1559.

[36] Theodoros D. Vrionis, Xanthi I. Koutiva, Nicholas A. Vovos, "A Genetic Algorithm-Based Low Voltage Ride-Through Control Strategy for Grid Connected Doubly Fed Induction Wind Generators", IEEE Transactions on Power Systems, Vol. 29, 2014, pp. 1325-1334.

[37] John Mc Call, "Genetic algorithms for modelling and optimization", Journal of Computational and Applied Mathematics, Vol. 184, 2005, pp. 205-222.

[38] Daiki Min, Jong-hyun Ryu, Dong Gu Choi, "A longterm capacity expansion planning model for an electric power system integrating large-size renewable energy technologies", Computers and Operations Research, Vol. 96, 2018, pp. 244-255.

[39] C. Roldan, A. A. Sanchez de la Nieta, R. Garcia-Bertrand, R. Minguez, "Robust dynamic transmission and renewable generation expansion planning: Walking towards sustainable systems", International Journal of Electrical Power and Energy Systems, Vol. 96, 2018, pp. 52-63.

[40] Angela Flores-Quiroz, Rodrigo Palma-Behnke, Golbon Zakeri, Rodrigo Moreno, “A column generation approach for solving generation expansion planning problems with high renewable energy penetration", Electric Power Systems Research, Vol. 136, 2016, pp. 232-241.
[41] Junhao Zhang, Pingi Xia, "An improved PSO algorithm for parameter identification of nonlinear dynamic hysteretic models", Journal of Sound and Vibration, Vol. 389, 2017, pp. 153-167.

[42] Kennedy, R. Eberhart, "Particle swarm optimization", Proceedings of the IEEE International Conference on Neural Networks, Perth, WA, Australia, 27 November-1 December 1995, pp. 1942-1948.

[43] Y. X. Jin, H. Z. Cheng, J. Y. Yan, L. Zhang, “New discrete method for particle swarm optimization and its application in transmission network expansion planning", Electric Power Systems Research, Vol. 77, 2007, pp. 227-233.

[44] Ping Ren, Nan Li, "Optimal expansion planning of high-voltage transmission network using the composite particle swarm optimization", Proceedings of the International Conference on Artificial Intelligence and Computational Intelligence, Sanya, China, 23-24 October 2010, pp. 170-173.

[45] Saeid Jalilzadeh, Ali Kimiyaghalam, Amir Bagheri, Ahmad Ashouri, "Application of IDPSO Approach for TNEP Problem Considering the Loss and Uncertainty in load growth", Proceedings of the International Congress on Ultra Modern Telecommunications and Control Systems and Workshops, Moscow, Russia, 18-20 October 2010, pp. 232-240.

[46] Baris Kocer, "Bollinger bands approach on boosting $A B C$ algorithm and its variants", Applied Soft Computing, Vol. 49, 2016, pp. 292-312.

[47] Kanendra Naidu, Hazlie Mokhlis, Ab Halim Abu Bakar, Vladimir Terzijia, "Performance investigation of $A B C$ algorithm in multi-area power system with multiple interconnected generators", Applied Soft Computing, Vol. 57, 2017, pp. 436-451.

[48] Hui Wang, Wenjun Wang, Xinyu Zhou, Hui Sun, Zhihua Cui, "Firefly algorithm with neighborhood attraction", Information Sciences, Vol. 382, 2017, pp. 374-387.

[49] Abdollah Rastgou, Jamal Moshtagh, "Application of Firefly Algorithm for multi-stage Transmission Expansion Planning with Adequacy-security considerations in Deregulated Environments", Applied Soft Computing, Vol. 41, 2016, pp. 373-389. 
[50] J. Qui, J. Zhao, Z.Y. Dong, "Probabilistic transmission expansion planning for increasing wind power penetration", IET Renewable Power Generation, Vol. 11, 2017, pp. 837-845.

[51] S. Majumder, R. M. Shereef, S. A. Khaparde, "Twostage algorithm for efficient transmission expansion planning with renewable energy resources", IET Renewable Power Generation, Vol. 11, 2017, pp. 320-329.

[52] S. Dehghan, N. Amjady, "Robust Transmission and Energy Storage Expansion Planning in Wind FarmIntegrated Power Systems Considering Transmission Switching", IEEE Transactions on Sustainable Energy, Vol. 7, 2016, 1981, pp. 765-774.

[53] Roy Z. Wu, P. Zeng, X. P. Zhang, "Two-stage stochastic dual dynamic programming for transmis- sion expansion planning with significant renewable generation and N-k criterion", CSEE Journal of Power and Energy Systems, Vol. 2, 2016, pp. 3-10.

[54] I. Fuchs, S. Voller, T. Gjengedal, “Improved method for integrating renewable energy sources in to the power system of Northern Europe: Transmission expansion planning for wind power integration", Proceedings of $10^{\text {th }}$ International Conference on Environment and Electrical Engineering, Rome, Italy, 8-11 May 2011, pp. 1-4.

[55] Jin Lin, Lin Cheng, Yao Chang, Kai Zhang, Guangyi Liu, "Reliability based power systems planning and operation with wind power integration: A review to models, algorithms and applications", Renewable and Sustainable Energy Reviews, Vol. 31, 2014, pp. 921-934. 